\title{
Asymmetrical blood flow in the temporal lobe in the Charles Bonnet syndrome: serial neuroimaging study
}

\author{
N. Adachi, ${ }^{1}$ M. Nagayama, ${ }^{1,2}$ K. Anami, ${ }^{1}$ K. Arima ${ }^{1}$ and H. Matsuda ${ }^{1}$ \\ ${ }^{1}$ National Center Hospital for Mental, Nervous, and Muscular Disorders, National Center of \\ Neurology and Psychiatry, 4-1-1 Ogawahigashi, Kodaira, Tokyo 187, and ${ }^{2}$ Department of \\ Psychiatry, Japanese Red Cross Medical Center, Tokyo, Japan
}

Correspondence to: $N$. Adachi at above address

\begin{abstract}
Clinical features and results of neuroimagings of an 86 year old woman with the Charles Bonnet syndrome are reported. She had become completely blind bilaterally due to cataracts and glaucoma. Shortly after an operation for cataracts, she developed visual hallucinations which lasted for 22 years. She had no deterioration of intelligence. Computed tomography (CT) and magnetic resonance imaging (MRI) showed moderate generalized atrophy, particularly of the temporal lobes. A serial single photon emission computed tomography (SPECT) study during visual hallucinations demonstrated hyperperfusion in the left temporal region and the basal ganglia and hypoperfusion in the right temporal region. These findings suggest that asymmetrical blood flow, particularly in the temporal regions, may be correlated with visual hallucination in the Charles Bonnet syndrome.
\end{abstract}

Keywords: Charles Bonnet syndrome - Neuroimaging - SPECT - Temporal lobe - Visual hallucination

\section{INTRODUCTION}

Visual hallucination in elderly sane people with eye disease was defined as the Charles Bonnet syndrome by de Morsier (1936). As this syndrome is a rare phenomenon, there are few reports concerning its neuroimaging (White, 1980; Olbrich et al., 1987; Rosembaum et al., 1987; Podoll et al., 1989; Siatkowski et al., 1990). We report, therefore, the computed tomography (CT), magnetic resonance imaging (MRI) and single photon emission computed tomography (SPECT) findings of an 86 year old woman who had experienced visual hallucinations for 22 years.

\section{CASE REPORT}

An 86 year old woman had become completely blind after operations for bilateral cataracts and secondary angle-closure glaucoma and as a consequence developed the Charles Bonnet syndrome (visual hallucinations associated with acquired blindness). These hallucinations had been present for 22 years since the operation and took the form of the face of a Kabuki actor or the imaginary animal "shishi". She had a familiar feeling about the hallucinations and knew they were not real (pseudohallucination).

After suffering from a common cold, at the age of 83 years, the frequency of her hallucinations in-

(C) 1994 Rapid Communications of Oxford Ltd creased and a secondary delusion, that her daughter loved shishi, arose. As the delusion interfered with her daily life, she was admitted to the National Center Hospital for Mental, Nervous and Muscular Disorders.

On admission, although visual hallucinations were present almost all day long, her intelligence remained quite intact (full score of Mini-mental State except for the subtests requiring visual function). Neurological examinations showed no abnormality. Routine hematologic examination and serum and cerebrospinal fluid examinations were unremarkable. The hallucinations were basically resistant to therapy, particularly benzodiazepines and major tranquillizers (i.e. haloperidol and sulpiride), which easily caused confusion, but tiapride reduced the frequency and severity of the hallucinations.

A brain CT scan showed moderate generalized atrophy, particularly of the temporal lobes. MRI showed bilateral symmetrical atrophy of the temporal lobes and enlargement of the calcarine fissures (Figure 1). She underwent a serial SPECT study using ${ }^{99 m}$ Tc-HMPAO and ${ }^{123}$ I-IMP during hallucinations at ages 83,84 and 86 , which were performed at $10.00 \mathrm{~h}$ under tiapride treatment $(200 \mathrm{mg} /$ day). These showed left temporal hyperperfusion in both mesial structures (hippocampus and parahippocampal 


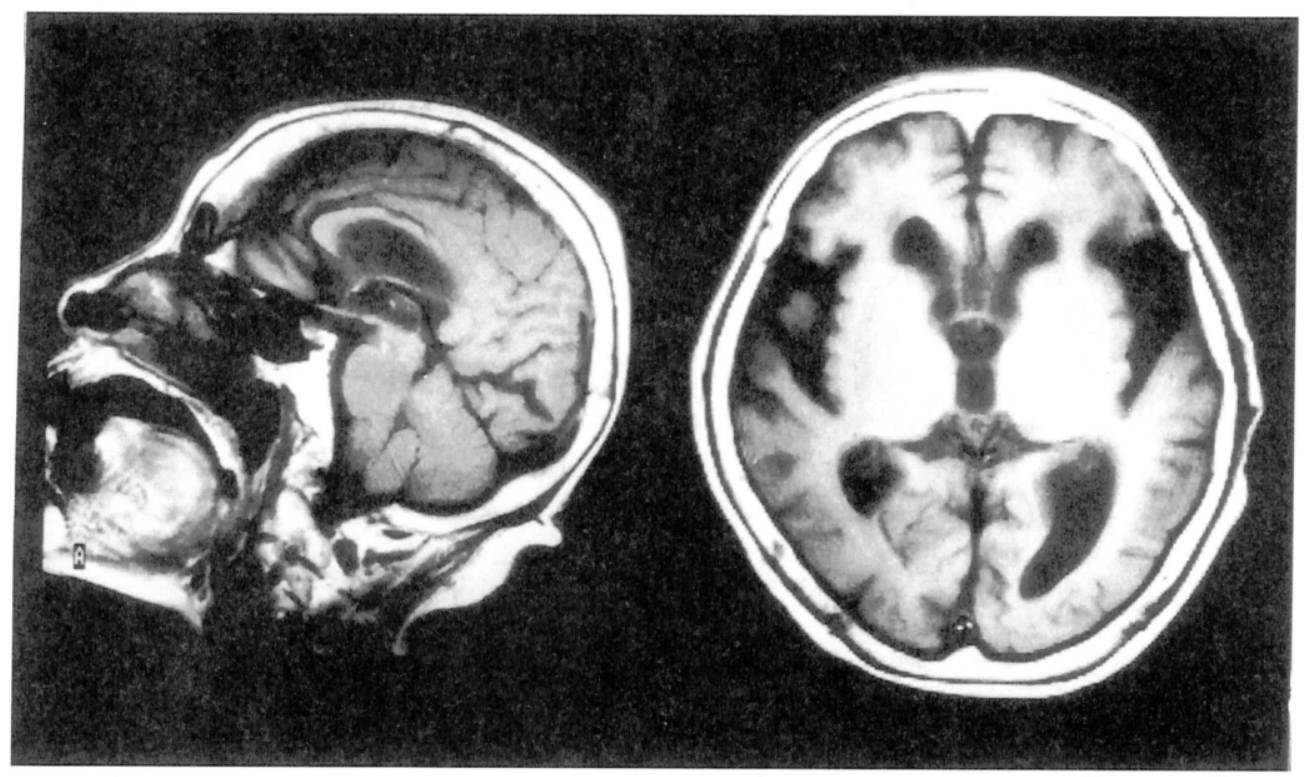

FIG. 1. MRI study performed at age 86 during visual hallucination. Left: T1-weighted sagital images with $\mathrm{TE}=19 \mathrm{~ms}$ and $\mathrm{TR}=550 \mathrm{~ms}$ demonstrated enlargement of the calcarine fissure. Right: T1-weighted transaxial images with $\mathrm{TE}=19 \mathrm{~ms}$ and $\mathrm{TR}=550 \mathrm{~ms}$ demonstrated diffuse cortical and subcortical atrophy (more in the temporal lobes bilaterally).

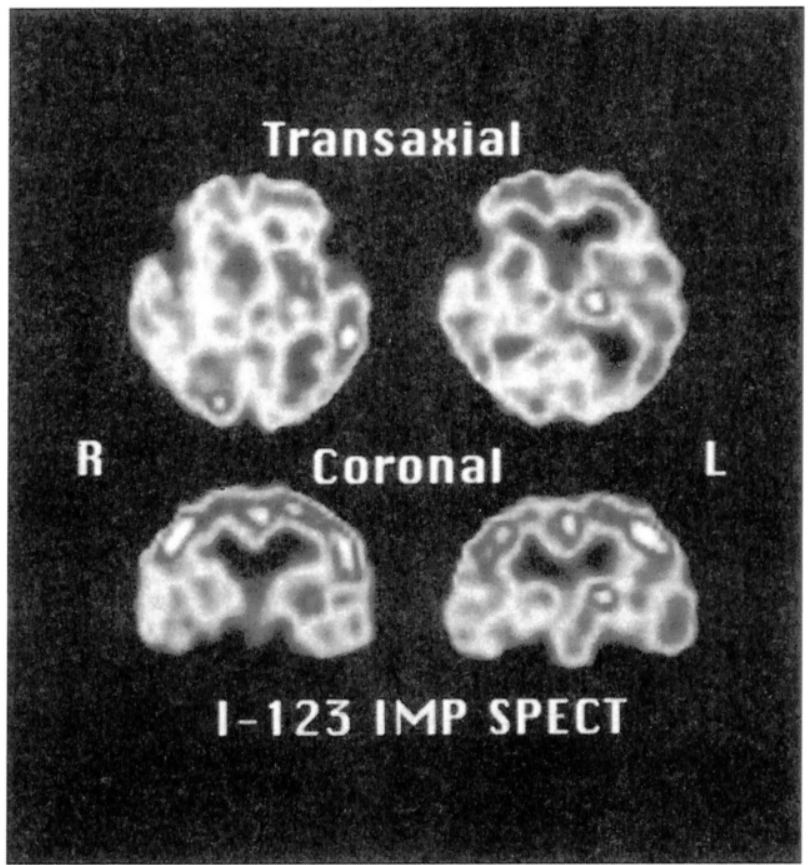

FIG. 2. SPECT study using IMP at the age of 86 years during visual hallucination. Transaxial section disclosed hyperperfusion in the left temporal lobe (both mesial and lateral structures), thalamus, and corpus striatum. Appearances in the occipital lobe were unremarkable. Coronal section disclosed hypoperfusion in the right mesial temporal structures.

gyrus) and the lateral cortex (middle and inferotemporal visual areas), despite the known temporal lobe atrophy (Figure 2). Hyperperfusion was also seen in the left thalamus and corpus striatum. There was no marked change in the occipital region on either side (areas 17, 18 and 19). Electroencephalography under the same conditions showed occipital dominant symmetrical $8-9 \mathrm{~Hz}$ alpha rhythm and no epileptic activity. Short latency somatosensory evoked potential (SSEP) by median nerve stimulation and auditory brainstem responses (ABR) showed normal peak latencies and voltages. Visual evoked potentials (VEP) by flashing light showed no response.

\section{DISCUSSION}

Since de Morsier (1936) defined the Charles Bonnet syndrome, approximately 60 cases have been reported (Gold and Ravins, 1989; Podoll et al., 1989; Siatkowski et al., 1990; Adachi and Nagayama, 1992). Few authors, however have carried out neuroimaging other than with CT (White, 1980; Olbrich et al., 1987; Rosembaum et al., 1987; Gold and Ravins 1989; Podoll et al., 1989; Siatkowski et al., 1990). In the case reported here, the CT and MRI studies disclosed atrophy of the temporal lobes. In their review Podoll et al. (1989) cited no report of a specific change on CT except for mild atrophy, probably age-related. No previous studies have reported findings on functional imaging in this syndrome. In our series of SPECT studies, asymmetrical blood flow patterns in the temporal regions (hyperperfusion on

98 Behavioural Neurology . Vol 7 . 1994 
the left and hypoperfusion on the right) were observed constantly in spite of the symmetrical cerebral atrophy seen on CT and MRI. The enlargement of the calcarine fissure on MRI did not accord with the findings in the serial SPECT study.

The etiology of the Charles Bonnet syndrome remains controversial. There are four main hypotheses which relate to eye pathology (Damas-Mora et al., 1982), release phenomenon caused by sensory deprivation (Cogan, 1973; Olbrich et al., 1987; Siatkowski et al., 1990), cortical irritation (Berrios and Brook, 1984; Rosembaum et al., 1987), and psychic reaction to blindness or bereavement (Alroe and McIntyre, 1983). It is likely that hallucination depends more upon a combination of several factors than a single factor (Berrios and Brook, 1984; Adachi and Nagayama, 1992). Our findings suggest that changes in the cerebral circulation, particularly in the temporal regions, correlate with visual hallucinations, although it is difficult to determine whether a specific area of the left temporal lobe is responsible for the generation of these formed hallucinations. One possibility is that they arise from an interaction between the hippocampus and the middle and inferotemporal visual areas. Pandya and Yeterian (1985) stated that the visual association cortex has paralimbic connections. Clear formed visual hallucinations are recognized to follow electrical stimulation of the temporal lobe (Gloor et al., 1982). However, in our case, the hallucinations were distinguished from epileptic phenomena by the normal EEG. Nevertheless cortical "excitation" of the temporal lobe was observed. Some clinical reports (Cohen et al., 1992) also indicate that an organic disturbance of the temporal lobe might cause a complex visual hallucination in the absence of epileptic discharges. By contrast, visual hallucinations arising from occipital lobe dysfunction usually comprise simple and elementary forms, such as stars and flashing lights.

In conclusion, we suggest that cortical and/or subcortical dysfunction of the temporal lobes is the likely cause of visual hallucinations in the Charles Bonnet syndrome. More detailed neuroimaging studies are required to clarify the functional anatomical pathways involved in the pathogenesis of the syndrome.

\section{REFERENCES}

Adachi N and Nagayama M (1992) A case of Charles Bonnet syndrome (in Japanese). Seishin-Igaku, 34, 83-87.

Alroe CJ and McIntyre JNM (1983) Visual hallucinations: The Charles Bonnet syndrome and bereavement. Medical Journal of Australia, 2, 674-675.

Berrios GE and Brook P (1984) Visual hallucinations and sensory delusions in the elderly. British Journal of Psychiatry, 144, 662-664.

Cogan DG (1973) Visual hallucinations as release phenomena. Graefe's Archives of Clinical and Experimental Ophthalmology, 188, 139-150.

Cohen L, Verstichel P and Pierrot-Deseilligny C (1992) Hallucinatory vision of a familiar face following right temporal hemorrhage. Neurology, 42, 2052.

Damas-Mora J, Skelton-Robinson $\mathbf{M}$ and Jenner FA (1982) The Charles Bonnet syndrome in perspective. Psychological Medicine, 12, 251-261.

de Morsier G (1936) Les automatism visuels: Hallucinations visuells retrochiasmatiques. Schweizerische Medizinische Wochenschrift, 29, 700-703.

Gloor P, Olivier A, Quesney LF, Andermann F and Horowitz $S$ (1982) The role of the limbic system in experimental phenomena of temporal lobe epilepsy. Annals of Neurology, 12, 129-144.

Gold K and Ravins PV (1989) Isolated visual hallucinations and the Charles Bonnet syndrome: A review of literature and presentation of six cases. Compr. Psychiatry, 30, 9098.

Olbrich HM, Engelmeier MP, Pauleikhoff D and Waubke $T$ (1987) Visual hallucinations in ophthalmology. Graefe's Archives of Clinical and Experimental Ophthalmology, 225, 217-220.

Pandya DN and Yeterian EH (1985) Architecture and connections of cortical association areas. In: Cerebral Cortex, vol. 4. Association and Auditory Cortices (Eds A Peters and EG Jones), pp. 3-61. Plunum, New York.

Podoll K, Osterheider M and Noth J (1989) Das Charles Bonnet-syndrome. Fortschritte der Neurologie, Psychiatrie und ihrer Grenzgebiete, 57, 43-60.

Rosembaum F, Harati Y, Rolak L and Freedman M (1987) Visual hallucination in sane people: Charles Bonnet syndrome. Journal of the American Geriatric Society, 35, 66-68.

Siatkowski RM, Zimmer B and Rosenberg PR (1990) The Charles Bonnet syndrome. Visual perceptive dysfunction in sensory deprivation. Journal of Clinical Neuroophthalmology, 100, 215-218.

White NJ (1980) Complex visual hallucination in partial blindness due to eye disease. British Journal of Psychiatry, 136, 284-286.

(Received 2 January 1994; accepted as revised 16 June 1994) 


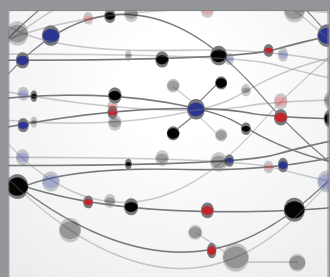

The Scientific World Journal
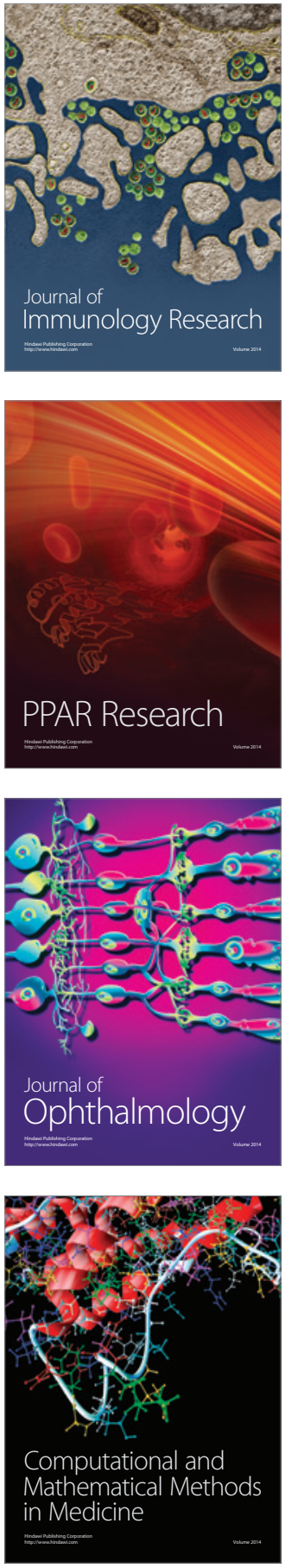

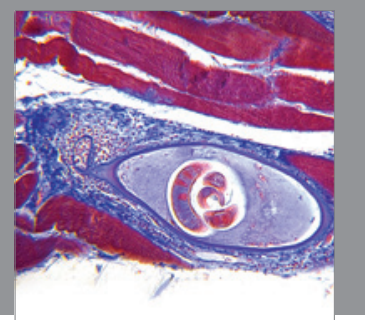

Gastroenterology

Research and Practice
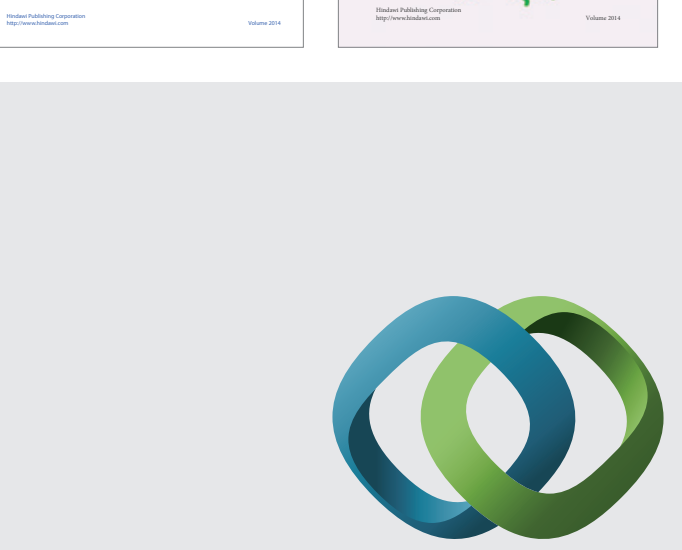

\section{Hindawi}

Submit your manuscripts at

http://www.hindawi.com
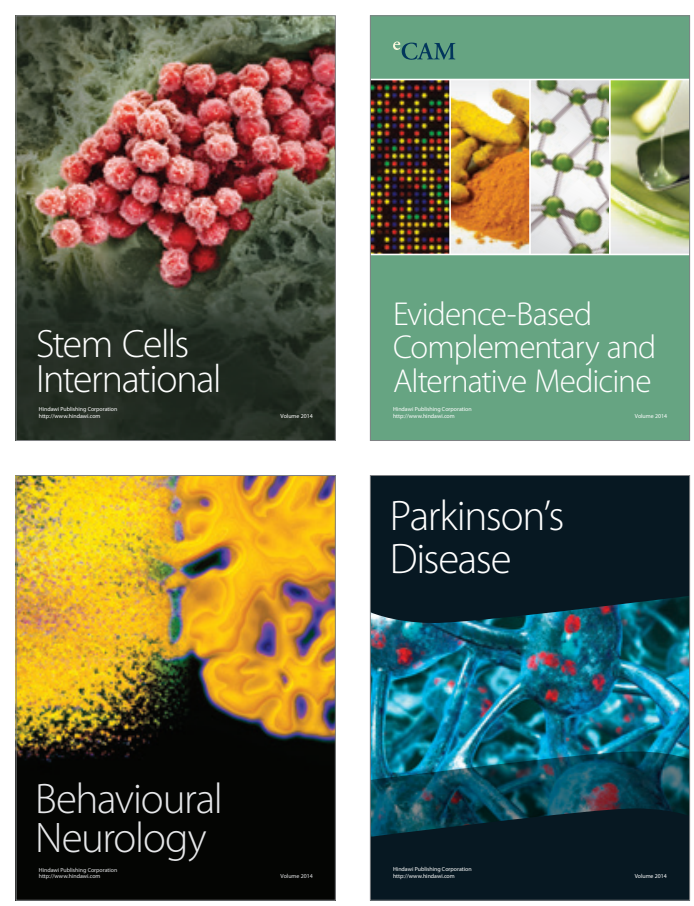

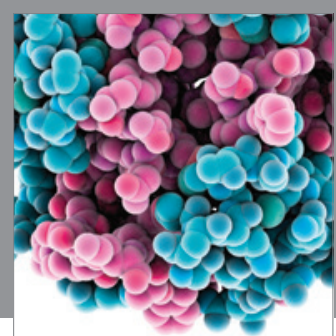

Journal of
Diabetes Research

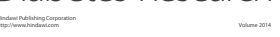

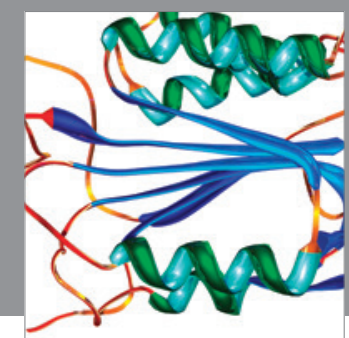

Disease Markers
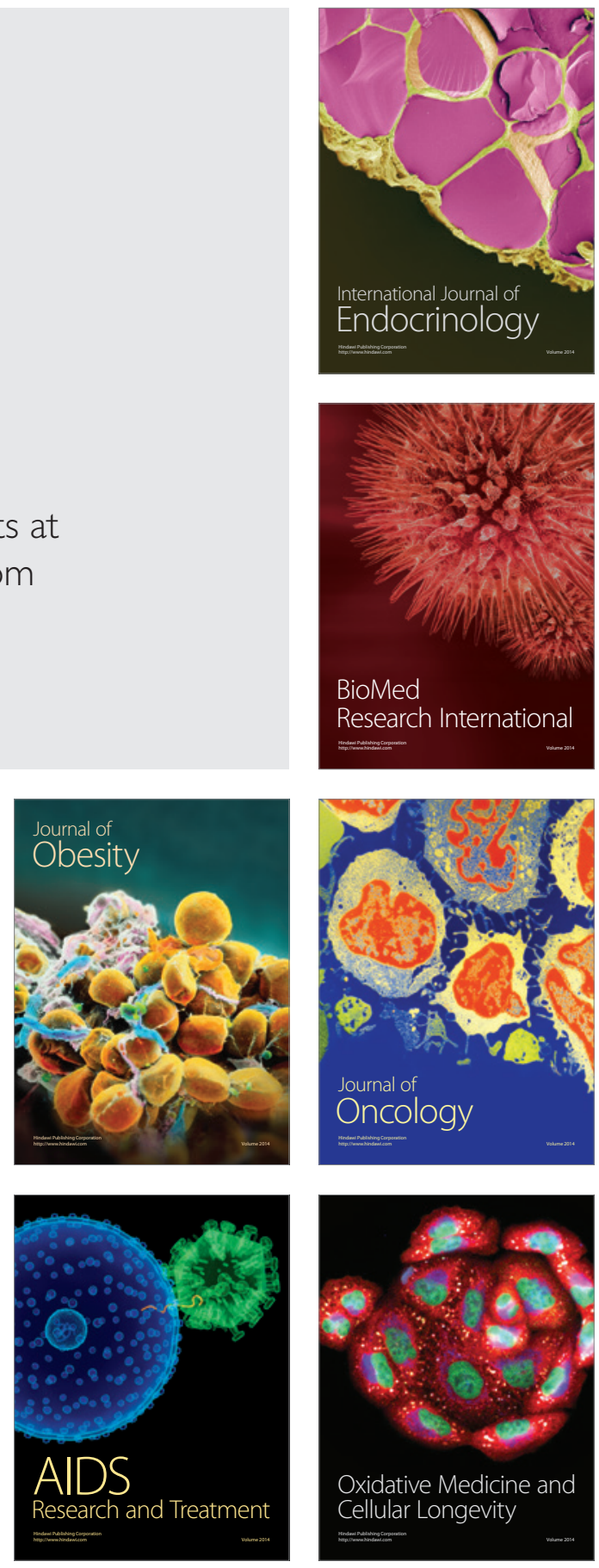